\author{
Military Technical College \\ Kobry Elkobbah, \\ Cairo, Egypt
}

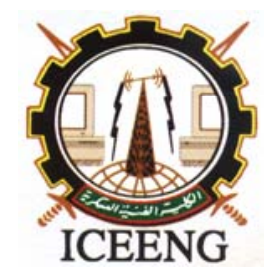
$5^{\text {th }}$ International Conference on Electrical Engineering
ICEENG 2006

\title{
STRAP DOWN INS ALIGNMENT USING NON-LINEAR MODEL FOR LARGE AZIMUTH MISALIGNMENT
}

\author{
Y. Z. Elhalwagy*, I. I. Arafa** and Ahmed M. Youssef***
}

Abstract:

In this paper a general non-linear psi-angle approach that dose not requires coarse alignment is presented. In the psi-angle model, the azimuth misalignment angle is assumed large. The attitude and velocity error models are also presented for strap down inertial navigation system (SDINS). Three different KALMAN filter algorithms are utilized based on the SDINS nonlinear error model are used to solve the non-linear data fusion problem. The proposed works are validated with a set of experimental results of stationary alignment and in-flight alignment using kinematics trajectory data to estimate all the parameters of inertial navigation system needed for the alignment and calibration.

Keywords: Inertial sensors, Inertial navigation system, strap down systems, alignment and error analysis

\footnotetext{
${ }^{*}$ Egyptian Armed Forces

${ }^{* *}$ Egyptian Armed Forces

*** Egyptian Armed Forces
} 


\section{Introduction}

The alignment of a strap down inertial navigation system (SDINS) determines the transformation matrix between body frame and navigation frame (local-level frame) [1-8]. The stationary initial alignment, which consists of a coarse alignment and a fine alignment, is usually performed when a vehicle is at rest. In this case, if low-grade sensors are used for cost reduction, it is virtually impossible to detect small attitude errors because its accuracy heavy depends on inertial sensors employed in alignments. For some applications, the coarse alignment is only performed or the initial attitude is directly obtained from other sources such as a stored attitude or a master inertial navigation system (INS) in order to reduce the initial alignment time [8]. In cases mentioned above, the initial attitude errors may be very large. Large attitude errors do not guarantee the accuracy and reliability of a system after beginning a navigation mode. Gimbaled INS (GINS) errors propagation models and strap down inertial navigation units have been subject of significant research during the past few years [1-4]. Two main approaches are used to derive these equations: psi-angle approach and perturbation approach $[2,4,7]$. The INS alignment and calibration tasks are usually based on these models.

In the literature, the initial orientation errors are assumed to be small, i.e. less than 5 degrees. The system can then be approximated with linear models due to the small angle assumption. To satisfy these requirements good quality gyros and external tilt and heading information has to be used. So far, few works attempted to model large angle errors to be considered, for example, large heading uncertainty of inertial measurement unit (IMU) orientation. In [11] and [6] an approximate extended psi-angle model with large heading misalignment is presented. It uses four states to describe the three-psi-angles. The model extension is very involved, and to the best of the author knowledge it has not been used in any practical application. Ref [5], introduced a Kalman filter mechanization for INS air star system. This approach uses two non-linear states to describe one heading angle. It still requires coarse ground alignment information within few degrees to estimate the wander angle. Ref [12] presents an INS error model considering large heading uncertainty and small tilt misalignment errors using a perturbation approach.

In this paper a general non-linear psi-angle approach is presented that dose not requires coarse alignment. In this model, the azimuth misalignment angle is assumed large. The attitude and velocity error models are also presented for strap down inertial navigation system (SINS). Three different Kalman filter algorithms are utilized based on the SDINS nonlinear error model and used to solve the non-linear data fusion problem. The presented model is validated with a set of experimental results of stationary alignment and in-flight alignment using kinematics trajectory data to estimate all the parameters of inertial navigation system needed for the alignment and calibration. Finally, the paper terminated with conclusions.

\section{Non-linear Error Model for SDINS}

The main objective of alignment process for a strap down system is to determine the direction cosine matrix $\mathrm{C}_{b}^{n}$, which defines the relationship between the inertial sensor axes and local geographic frame $[5,8]$. The measurements provided by the inertial sensors in body axes may be resolved into the local geographic frame using the current best estimated of the body attitude with respect to this frame. The resolved sensor measurements are then compared with the expected turn rate and accelerations to enable the direction cosine to be calculated correctly. In other words, SDINS uses mathematical platform rather than physical one (the mathematical platform is determined by $\mathrm{C}_{b}^{n}$ ). The attitude error equations may be 
determined by disturbance of nominal equation, but for GINS the attitude error equations could be figured out by using the actual error angles [8].

Let, the misalignment angle $\psi$ between platform frame (p-frame) and navigation frame (n-frame) is defined as:

$$
\psi=\left[\begin{array}{lll}
\psi_{x} & \psi_{y} & \psi_{z}
\end{array}\right]^{T}
$$

and $\Psi$ is the skew symmetric matrix of psi-angle. Let the following symbols stand for:

$$
\mathrm{s}_{x}=\sin \left(\psi_{x}\right) ; \mathrm{s}_{y}=\sin \left(\psi_{y}\right) ; \mathrm{s}_{z}=\sin \left(\psi_{z}\right) ; \mathrm{c}_{x}=\cos \left(\psi_{x}\right) ; \mathrm{c}_{y}=\cos \left(\psi_{y}\right) ; \mathrm{c}_{z}=\cos \left(\psi_{z}\right) ;
$$

Then, the direction cosine matrix (DCM) from n-frame to p-frame can be defined as [7]:

$$
C_{n}^{p}=\left[\begin{array}{ccc}
c_{y} c_{z}-s_{y} s_{x} s_{z} & c_{y} s_{z}+s_{y} s_{x} c_{z} & -s_{y} c_{x} \\
-c_{x} s_{z} & c_{x} c_{z} & s_{x} \\
s_{y} c_{z}+c_{y} s_{x} s_{z} & s_{y} s_{z}-c_{y} s_{x} c_{z} & c_{y} c_{x}
\end{array}\right]
$$

\section{II.1 Velocity Error Model}

The SDINS true velocity error in navigation frame is given by $[7,8]$ :

$$
\boldsymbol{V}_{t}^{n}=C_{\mathrm{b}}^{\mathrm{n}} \boldsymbol{f}^{b}-\left(2 \Omega_{\mathrm{ie}}^{\mathrm{n}}+\omega_{\mathrm{en}}^{\mathrm{n}}\right) \times \boldsymbol{V}_{\mathrm{t}}^{\mathrm{n}}+\boldsymbol{g}^{\mathrm{n}}
$$

Where, $f^{b}, C_{b}^{n}$ are both the specific force in body frame and transformation matrix from body frame to navigation frame respectively. The SDINS solves the following velocity $\boldsymbol{V}_{c}^{c}$ in the computational frame:

$$
\boldsymbol{V}_{\mathrm{c}}^{\mathrm{c}}=\hat{C}_{\mathrm{b}}^{\mathrm{n}} \hat{\boldsymbol{f}}^{b}-\left(2 \hat{\Omega}_{i e}^{n}+\hat{\omega}_{e n}^{n}\right) \times \boldsymbol{V}_{\mathrm{c}}^{\mathrm{c}}+\boldsymbol{g}^{\mathrm{c}}
$$

Where, $\hat{\mathrm{C}}_{b}^{n}=\mathrm{C}_{b}^{p}$.

$\boldsymbol{g}^{\boldsymbol{c}}, \boldsymbol{g}^{\boldsymbol{n}}$ are the gravity vectors resolved in the computational frame and navigation frame respectively. $\mathbf{V}_{c}^{c}$ is the velocity vector resolved in the c-frame and can be calculated as:

$\boldsymbol{V}_{\mathrm{c}}^{\mathrm{c}}=\boldsymbol{V}_{\mathrm{t}}^{\mathrm{n}}+\delta \boldsymbol{V}$

$\hat{\mathbf{f}}^{b}$ is the estimated specific force in body frame that can be written as:

$$
\hat{f}^{b}=f^{b}+\nabla^{b}
$$

Where, $\nabla^{b}$ is the specific force error due to accelerometer bias in b-frame, and

$$
\begin{aligned}
& \hat{\Omega}_{i e}^{n}=\Omega_{i e}^{n}+\delta \Omega_{i e}^{n} \\
& \hat{\omega}_{e n}^{n}=\omega_{e n}^{n}+\delta \omega_{e n}^{n}
\end{aligned}
$$

Subtract Eq. (2) from Eq. (3) yields the SINS velocity error equation:

$$
\begin{aligned}
\delta \dot{V}= & \left(\mathrm{C}_{\mathrm{n}}^{\mathrm{p}}-I\right) \mathrm{C}_{\mathrm{b}}^{\mathrm{n}} f^{b}+\hat{\mathrm{C}}_{\mathrm{b}}^{\mathrm{n}} \nabla^{\mathrm{b}}-\left(2 \delta \Omega_{\mathrm{ie}}^{\mathrm{n}}+\delta \omega_{\mathrm{en}}^{\mathrm{n}}\right) \\
& \times V_{\mathrm{t}}^{\mathrm{n}}-\left(2 \Omega_{\mathrm{ie}}^{\mathrm{n}}+\omega_{\mathrm{en}}^{\mathrm{n}}\right) \times \delta V+\delta g^{\mathrm{n}}
\end{aligned}
$$

The above equation as a function of estimated specific force $\hat{\mathrm{f}}^{b}$ could be written as:

$$
\begin{aligned}
\delta \dot{V}= & \left(I-\mathrm{C}_{\mathrm{p}}^{\mathrm{n}}\right) \hat{\mathrm{C}}_{\mathrm{b}}^{\mathrm{n}} \hat{f}^{\mathrm{b}}+\mathrm{C}_{\mathrm{b}}^{\mathrm{n}} \nabla^{\mathrm{b}}-\left(2 \delta \Omega_{\mathrm{ie}}^{\mathrm{n}}+\delta \omega_{\mathrm{en}}^{\mathrm{n}}\right) \\
& \times V_{\mathrm{t}}^{\mathrm{n}}-\left(2 \Omega_{\mathrm{ie}}^{\mathrm{n}}+\omega_{\mathrm{en}}^{\mathrm{n}}\right) \times \delta V+\delta g^{\mathrm{n}}
\end{aligned}
$$

Let $\hat{\mathrm{C}}_{b}^{n} \hat{f}^{b}=\hat{f}^{p}, \mathrm{C}_{\mathrm{b}}^{\mathrm{n}} \nabla^{b}=\mathrm{C}_{p}^{n} \nabla^{p}$ and when the attitude error is small then, the transformation matrix $\mathrm{C}_{n}^{p}$ can be expresses as:

$$
\mathrm{C}_{\mathrm{n}}^{\mathrm{p}}=I-[\Phi \times]
$$


When the attitude error is small, as we know from Eq. (6) the small disturbance equations can be written as follows:

$$
\begin{aligned}
\delta \dot{V}= & \left(\mathrm{C}_{\mathrm{b}}^{\mathrm{n}} f^{b}\right) \times \Phi-\left(2 \delta \Omega_{\mathrm{ie}}^{\mathrm{n}}+\delta \omega_{\mathrm{en}}^{\mathrm{n}}\right) \\
& \times V_{\mathrm{t}}^{\mathrm{n}}-\left(2 \Omega_{\mathrm{ie}}^{\mathrm{n}}+\omega_{e n}^{\mathrm{n}}\right) \times \delta V+\hat{\mathrm{C}}_{\mathrm{b}}^{\mathrm{n}} \nabla^{b}+\delta g^{\mathrm{n}}
\end{aligned}
$$

\section{II.2 Attitude Error Model}

The psi-angle model for small angle errors was presented before in [13]. This paper presents a new psi- angle model that can be used with large angle errors.

The true transformation matrix $\mathrm{C}_{b}^{n}$ can be written as:

$$
\dot{C}_{b}^{n}=C_{b}^{n}\left[\omega_{i b}^{b} \times\right]-\left[\omega_{i n}^{n} \times\right] C_{b}^{n}
$$

where, $\left[\omega_{i b}^{b} \times\right]$ is the skew symmetric matrix and $\omega_{i b}^{b}$ is the computed angular velocity of body with respect to inertial frame.

The matrix $C_{b}^{p}$ is obtained using measured gyro rates $\hat{\omega}_{i b}^{b}$ provided by the IMU:

$$
\boldsymbol{C}_{\mathrm{b}}^{\mathrm{p}}=C_{b}^{p}\left[\hat{\omega}_{i b}^{b} \times\right]-\left[\hat{\omega}_{i n}^{n} \times\right] C_{b}^{p}
$$

Where, $\mathrm{C}_{b}^{p}$ is the transformation matrix from body to platform frame (or written as $\hat{\mathrm{C}}_{\mathrm{b}}^{\mathrm{n}}$ ); $\hat{\omega}_{i n}^{n}=\omega_{i n}^{n}+\delta \omega_{i n}^{n}$ where $\omega_{i n}^{n}, \delta \omega_{i n}^{n}$ is the true angular velocity and angular velocity error of navigation frame with respect to inertial frame.

$\hat{\omega}_{i b}^{b}$ contains gyros drift errors $\delta \omega_{i b}^{b}$ that can be large, specially when working with low cost IMU.

$$
\hat{\omega}_{i b}^{b}=\omega_{i b}^{b}+\delta \omega_{i b}^{b}
$$

Let $\Delta \mathrm{C}=\mathrm{C}_{\mathrm{b}}^{\mathrm{p}}-\mathrm{C}_{\mathrm{b}}^{\mathrm{n}}$, then

$$
\Delta \mathrm{C}=\mathrm{C}_{\mathrm{b}}^{\mathrm{p}}-\mathrm{C}_{\mathrm{b}}^{\mathrm{n}}=\mathrm{C}_{\mathrm{b}}^{\mathrm{p}}-\mathrm{C}_{\mathrm{p}}^{\mathrm{n}} \mathrm{C}_{\mathrm{b}}^{\mathrm{p}}=\left(I-\mathrm{C}_{\mathrm{p}}^{\mathrm{n}}\right) \mathrm{C}_{\mathrm{b}}^{\mathrm{p}}
$$

$\Delta \mathrm{C}$ can be derived from Eqs. $(9,10)$ :

$$
\begin{aligned}
\Delta \dot{\mathrm{C}}= & \left(I-\mathrm{C}_{p}^{n}\right) \mathrm{C}_{\mathrm{b}}^{\mathrm{p}}-\mathrm{C}_{\mathrm{p}}^{\mathrm{n}} \mathrm{C}_{\mathrm{b}}^{\mathrm{p}} \\
= & \mathrm{C}_{\mathrm{b}}^{\mathrm{p}}\left[\hat{\omega}_{\mathrm{ib}}^{\mathrm{b}} \times\right]-\left[\hat{\omega}_{\mathrm{in}}^{\mathrm{n}} \times\right] \mathrm{C}_{\mathrm{b}}^{\mathrm{p}}-\mathrm{C}_{\mathrm{p}}^{\mathrm{n}} \mathrm{C}_{\mathrm{b}}^{\mathrm{p}}\left[\hat{\omega}_{\mathrm{ib}}^{\mathrm{b}} \times\right]+ \\
& \mathrm{C}_{\mathrm{p}}^{\mathrm{n}}\left[\hat{\omega}_{\mathrm{in}}^{\mathrm{n}} \times\right] \mathrm{C}_{\mathrm{p}}^{\mathrm{n}} \mathrm{C}_{\mathrm{b}}^{\mathrm{p}}-\mathrm{C}_{p}^{n} \mathrm{C}_{\mathrm{b}}^{\mathrm{p}}
\end{aligned}
$$

$\Delta \dot{\mathrm{C}}$ can also obtained from $\Delta \mathrm{C}=\mathrm{C}_{\mathrm{b}}^{\mathrm{p}}-\mathrm{C}_{\mathrm{b}}^{\mathrm{n}}$

$$
\begin{aligned}
\Delta \dot{\mathrm{C}}= & \mathrm{C}_{b}^{p}-\mathrm{C}_{\mathrm{b}}^{\mathrm{n}} \\
= & \mathrm{C}_{\mathrm{b}}^{\mathrm{p}}\left[\hat{\omega}_{\mathrm{ib}}^{\mathrm{b}} \times\right]-\left[\hat{\omega}_{\mathrm{in}}^{\mathrm{n}} \times\right] \mathrm{C}_{\mathrm{b}}^{\mathrm{n}}-\mathrm{C}_{\mathrm{p}}^{\mathrm{n}} \mathrm{C}_{\mathrm{b}}^{\mathrm{p}}\left[\omega_{\mathrm{ib}}^{\mathrm{b}} \times\right] \\
& -\left[\omega_{\mathrm{in}}^{\mathrm{n}} \times\right] \mathrm{C}_{\mathrm{p}}^{\mathrm{n}} \mathrm{C}_{b}^{p}
\end{aligned}
$$

From Eqs. $(11,12)$, we get:

$$
\mathrm{C}_{\mathrm{p}}^{\mathrm{n}} \mathrm{C}_{\mathrm{b}}^{\mathrm{p}}\left[\delta \omega_{\mathrm{ib}}^{\mathrm{b}} \times\right]-\mathrm{C}_{\mathrm{p}}^{\mathrm{n}}\left[\hat{\omega}_{\mathrm{in}}^{\mathrm{n}} \times\right] \mathrm{C}_{\mathrm{b}}^{\mathrm{p}}+\mathrm{C}_{p}^{n} \mathrm{C}_{\mathrm{b}}^{\mathrm{p}}+\left[\omega_{\mathrm{in}}^{\mathrm{n}} \times\right] \mathrm{C}_{\mathrm{p}}^{\mathrm{n}} \mathrm{C}_{\mathrm{b}}^{\mathrm{p}}=0
$$

Right multiply $\mathrm{C}_{\mathrm{p}}^{\mathrm{b}}$ to the above equation yield:

$$
\mathrm{C}_{\mathrm{p}}^{\mathrm{n}}+\mathrm{C}_{\mathrm{p}}^{\mathrm{n}} \mathrm{C}_{\mathrm{b}}^{\mathrm{p}}\left[\delta \omega_{\mathrm{ib}}^{\mathrm{b}} \times\right] \mathrm{C}_{\mathrm{p}}^{\mathrm{b}}-\mathrm{C}_{\mathrm{p}}^{\mathrm{n}}\left[\hat{\omega}_{\mathrm{in}}^{\mathrm{n}} \times\right]+\left[\omega_{\mathrm{in}}^{\mathrm{n}} \times\right] \mathrm{C}_{\mathrm{p}}^{\mathrm{n}}=0
$$

It can be proved that 


$$
\left[\delta \omega_{\mathrm{ib}}^{\mathrm{p}} \times\right]=\mathrm{C}_{\mathrm{b}}^{\mathrm{p}}\left[\delta \omega_{\mathrm{ib}}^{\mathrm{b}} \times\right] \mathrm{C}_{\mathrm{p}}^{\mathrm{b}}=\mathrm{C}_{\mathrm{n}}^{\mathrm{p}}\left[\delta \omega_{\mathrm{ib}}^{\mathrm{n}} \times\right] \mathrm{C}_{\mathrm{p}}^{\mathrm{n}}
$$

and; $\quad \mathrm{C}_{\mathrm{p}}^{\mathrm{n}}\left[\delta \omega_{\mathrm{ib}}^{\mathrm{p}} \times\right]=\left[\delta \omega_{\mathrm{ib}}^{\mathrm{n}} \times\right] \mathrm{C}_{\mathrm{p}}^{\mathrm{n}}$.

Consequently, Eq. (15) can be simplified as:

$$
\mathrm{C}_{\mathrm{p}}^{\mathrm{n}}+\left[\delta \omega_{\mathrm{ib}}^{\mathrm{n}} \times\right] \mathrm{C}_{\mathrm{p}}^{\mathrm{n}}-\mathrm{C}_{\mathrm{p}}^{\mathrm{n}}\left[\hat{\omega}_{\mathrm{in}}^{\mathrm{n}} \times\right]+\left[\omega_{\mathrm{in}}^{\mathrm{n}} \times\right] \mathrm{C}_{\mathrm{p}}^{\mathrm{n}}=0
$$

Replacing $\mathrm{C}_{p}^{n}=\mathrm{C}_{\mathrm{p}}^{\mathrm{n}}\left[\omega_{\mathrm{np}}^{\mathrm{p}} \times\right]$ and left multiplying $\mathrm{C}_{\mathrm{n}}^{\mathrm{p}}$ to Eq. (16):

$$
\left[\omega_{\mathrm{np}}^{\mathrm{p}} \times\right]+\mathrm{C}_{\mathrm{n}}^{\mathrm{p}}\left[\delta \omega_{\mathrm{ib}}^{\mathrm{n}} \times\right] \mathrm{C}_{\mathrm{p}}^{\mathrm{n}}-\left[\hat{\omega}_{\mathrm{in}}^{\mathrm{n}} \times\right]+\mathrm{C}_{\mathrm{n}}^{\mathrm{p}}\left[\omega_{\mathrm{in}}^{\mathrm{n}} \times\right] \mathrm{C}_{\mathrm{p}}^{\mathrm{n}}=0
$$

we can notice that: $\mathrm{C}_{\mathrm{n}}^{\mathrm{p}}\left[\delta \omega_{\mathrm{ib}}^{\mathrm{n}} \times\right] \mathrm{C}_{\mathrm{p}}^{\mathrm{n}}=\left[\delta \omega_{\mathrm{ib}}^{\mathrm{p}} \times\right]$ and $\mathrm{C}_{\mathrm{n}}^{\mathrm{p}}\left[\omega_{\mathrm{in}}^{\mathrm{n}} \times\right] \mathrm{C}_{\mathrm{p}}^{\mathrm{n}}=\left[\omega_{\mathrm{in}}^{\mathrm{p}} \times\right]$, then Eq. (17) changes to:

$$
\left[\omega_{\mathrm{np}}^{\mathrm{p}} \times\right]+\left[\delta \omega_{\mathrm{ib}}^{\mathrm{p}} \times\right]-\left[\hat{\omega}_{\mathrm{in}}^{\mathrm{n}} \times\right]+\left[\omega_{\mathrm{in}}^{\mathrm{p}} \times\right]=0
$$

The terms $\left(\left[\omega_{\mathrm{np}}^{\mathrm{p}} \times\right]+\left[\delta \omega_{\mathrm{ib}}^{\mathrm{p}} \times\right]-\left[\hat{\omega}_{\mathrm{in}}^{\mathrm{n}} \times\right]+\left[\omega_{\mathrm{in}}^{\mathrm{p}} \times\right]\right)$ are the sums of skew symmetric matrices of $\left(\omega_{\mathrm{np}}^{\mathrm{p}}+\delta \omega_{\mathrm{ib}}^{\mathrm{p}}-\hat{\omega}_{\mathrm{in}}^{\mathrm{n}}+\omega_{\mathrm{in}}^{\mathrm{p}}\right)$, and then Eq. (18) can be written in the following form:

$$
\omega_{\mathrm{np}}^{\mathrm{p}}+\delta \omega_{\mathrm{ib}}^{\mathrm{p}}-\hat{\omega}_{\mathrm{in}}^{\mathrm{n}}+\omega_{\mathrm{in}}^{\mathrm{p}}=0
$$

With $\omega_{\text {in }}^{\mathrm{p}}=\mathrm{C}_{\mathrm{n}}^{\mathrm{p}} \omega_{\mathrm{in}}^{\mathrm{n}}$ and $\hat{\omega}_{\mathrm{in}}^{\mathrm{n}}=\omega_{\mathrm{in}}^{\mathrm{n}}+\delta \omega_{\mathrm{in}}^{\mathrm{n}}$ then Eq. (19) can be written as:

$$
\omega_{\mathrm{np}}^{\mathrm{p}}=\omega_{\mathrm{in}}^{\mathrm{n}}+\delta \omega_{\mathrm{in}}^{\mathrm{n}}-\mathrm{C}_{\mathrm{n}}^{\mathrm{p}} \omega_{\mathrm{in}}^{\mathrm{n}}-\mathrm{C}_{\mathrm{b}}^{\mathrm{p}} \delta \omega_{\mathrm{ib}}^{\mathrm{b}}=\left(I-\mathrm{C}_{\mathrm{n}}^{\mathrm{p}}\right) \omega_{\mathrm{in}}^{\mathrm{n}}+\delta \omega_{\mathrm{in}}^{\mathrm{n}}-\mathrm{C}_{\mathrm{b}}^{\mathrm{p}} \delta \omega_{\mathrm{ib}}^{\mathrm{b}}
$$

The three components of the Euler angle $\psi$ is not orthogonal so that, the relation between $\psi$ and $\omega_{\mathrm{np}}^{\mathrm{p}}$ can be written as [8]:

$$
\begin{aligned}
& \omega_{\mathrm{up}}^{\mathrm{P}}=\mathrm{C}_{\mathrm{u}}^{\mathrm{P}}\left[\begin{array}{c}
0 \\
0 \\
\psi_{z}
\end{array}\right]+\left[\begin{array}{ccc}
\mathrm{c}_{\mathrm{y}} & 0 & -\mathrm{s}_{\mathrm{y}} \\
0 & 1 & 0 \\
\mathrm{~s}_{\mathrm{y}} & 0 & \mathrm{c}_{\mathrm{y}}
\end{array}\right]\left[\begin{array}{ccc}
1 & 0 & 0 \\
0 & \mathrm{c}_{\mathrm{x}} & \mathrm{s}_{\mathrm{x}} \\
0 & -\mathrm{s}_{\mathrm{y}} & \mathrm{c}_{\mathrm{x}}
\end{array}\right]\left[\begin{array}{c}
\psi_{\mathrm{x}} \\
0 \\
0
\end{array}\right]+ \\
& {\left[\begin{array}{ccc}
\mathrm{c}_{\mathrm{y}} & 0 & -\mathrm{s}_{\mathrm{y}} \\
0 & 1 & 0 \\
\mathrm{~s}_{\mathrm{y}} & 0 & \mathrm{c}_{\mathrm{y}}
\end{array}\right]\left[\begin{array}{c}
0 \\
\dot{\psi}_{y} \\
0
\end{array}\right] }
\end{aligned}
$$

Using Eq. (1) yields:

$$
\omega_{\mathrm{np}}^{\mathrm{p}}=\left[\begin{array}{c}
\dot{\psi}_{\mathrm{x}} \mathrm{c}_{\mathrm{y}}-\dot{\psi}_{\mathrm{z}} \mathrm{s}_{\mathrm{y}} \mathrm{c}_{\mathrm{x}} \\
\dot{\psi}_{\mathrm{y}}+\dot{\psi}_{\mathrm{z}} \mathrm{s}_{\mathrm{x}} \\
\dot{\psi}_{\mathrm{x}} \mathrm{s}_{\mathrm{y}}+\dot{\psi}_{\mathrm{z}} \mathrm{c}_{\mathrm{y}} \mathrm{c}_{\mathrm{x}}
\end{array}\right]
$$

In alignment, if we consider small $\dot{\psi}$ and if the horizontal misalignment tilt angles $\psi_{x}, \psi_{y}$ are also small then, the angular velocity of platform with respect to navigation frame will be as:

$$
\omega_{\mathrm{np}}^{\mathrm{p}} \approx \dot{\psi}=\left[\begin{array}{c}
\dot{\psi}_{x} \\
\dot{\psi}_{y} \\
\dot{\psi}_{z}
\end{array}\right]
$$


When the horizontal misalignment angles $\psi_{x}, \psi_{y}$ are small as we know from Eq. (23) the SINS attitude error equation can be written as:

$$
\dot{\psi} \approx \omega_{\mathrm{np}}^{\mathrm{p}}=\left(I-\mathrm{C}_{\mathrm{n}}^{\mathrm{p}}\right) \omega_{\mathrm{in}}^{\mathrm{n}}+\delta \omega_{\mathrm{in}}^{\mathrm{n}}-\mathrm{C}_{\mathrm{b}}^{\mathrm{p}} \delta \omega_{\mathrm{ib}}^{\mathrm{b}}
$$

Then, Eq. (24) is the general psi-angle error model that can be used for small or large angle errors for SINS. When the three misalignment angles are small, then the attitude error model using Euler angle can be simplified to $\Psi$ angle as:

$$
\dot{\Psi}=\Psi \times \omega_{\mathrm{in}}^{\mathrm{n}}+\delta \omega_{\mathrm{in}}^{\mathrm{n}}-\mathrm{C}_{\mathrm{b}}^{\mathrm{p}} \delta \omega_{\mathrm{ib}}^{\mathrm{b}}
$$

\section{II.3 GINS Nonlinear Error Model}

If $\psi_{x}, \psi_{y}$ and $\psi_{z}$ are small, then $\mathrm{C}_{\mathrm{p}}^{\mathrm{n}}=\boldsymbol{I}+\Psi$.

$$
\mathrm{C}_{\mathrm{n}}^{\mathrm{p}}=\left[\begin{array}{ccc}
1 & \psi_{\mathrm{z}} & -\psi_{\mathrm{y}} \\
-\psi_{\mathrm{z}} & 1 & \psi_{\mathrm{x}} \\
\psi_{\mathrm{y}} & -\psi_{\mathrm{x}} & 1
\end{array}\right]
$$

and Eq. (20) can be converted to small psi-angle error model, which is widely used in many applications [7]:

$$
\dot{\psi}=\Psi \times \omega_{\mathrm{in}}^{\mathrm{n}}+\delta \omega_{\mathrm{in}}^{\mathrm{n}}-\mathrm{C}_{\mathrm{b}}^{\mathrm{p}} \delta \omega_{\mathrm{ib}}^{\mathrm{b}}
$$

In the case of large misalignment in heading and small misalignment in tilt angles, large $\psi_{z}$ and small $\psi_{x}$ and $\psi_{y}$ angles are resulted. With this assumption $\mathrm{C}_{\mathrm{n}}^{\mathrm{p}}$ can be approximated with [7]:

$$
\mathrm{C}_{\mathrm{n}}^{\mathrm{p}}=\left[\begin{array}{ccc}
\mathrm{c}_{\mathrm{z}} & \mathrm{s}_{\mathrm{z}} & -\psi_{\mathrm{y}} \\
-\mathrm{s}_{\mathrm{z}} & \mathrm{c}_{\mathrm{z}} & \psi_{\mathrm{x}} \\
\psi_{\mathrm{y}} \mathrm{c}_{\mathrm{z}}+\psi_{\mathrm{x}} \mathrm{s}_{\mathrm{z}} & \psi_{\mathrm{y}} \mathrm{s}_{\mathrm{z}}-\psi_{\mathrm{x}} \mathrm{c}_{\mathrm{z}} & 1
\end{array}\right]
$$

In the most INS alignment, the position error is not considered. So, the error angle between the computational frame and navigation frame caused by position error is equal zero. It means that, computational frame is aligned with navigation frame. So, the projection error of $\Omega_{i e}^{n}, \boldsymbol{g}$ is equal zero. i.e.,

$$
\delta \Omega_{i e}^{n}=0, \delta g^{n}=0
$$

Then, the platform velocity and attitude error equations model with large misalignment in Eq. $(4,24)$ can be written as:

$$
\begin{aligned}
& \delta \dot{V}=\left(\mathrm{C}_{\mathrm{n}}^{\mathrm{p}}-I\right) \mathrm{C}_{\mathrm{b}}^{\mathrm{n}} f^{b}+\hat{\mathrm{C}}_{\mathrm{b}}^{\mathrm{n}} \nabla^{b}-\delta \omega_{e n}^{\mathrm{n}} \times V_{\mathrm{t}}^{\mathrm{n}}-\left(2 \Omega_{\mathrm{ie}}^{\mathrm{n}}+\omega_{\mathrm{en}}^{\mathrm{n}}\right) \times \delta V \\
& \dot{\psi} \approx \omega_{\mathrm{np}}^{\mathrm{p}}=\left(I-\mathrm{C}_{\mathrm{n}}^{\mathrm{p}}\right) \omega_{\mathrm{in}}^{\mathrm{n}}+\delta \omega_{\mathrm{in}}^{\mathrm{n}}-\mathrm{C}_{\mathrm{b}}^{\mathrm{p}} \delta \omega_{\mathrm{ib}}^{\mathrm{b}}
\end{aligned}
$$

Where, $\delta \omega_{\mathrm{en}}^{\mathrm{n}}$ is the angular velocity error of navigation frame with respect to earth frame and it can be written as [9]:

$$
\delta \omega_{\mathrm{en}}^{\mathrm{n}}=\left[\begin{array}{ccc}
0 & -1 /\left(R_{M}+h\right) & 0 \\
1 /\left(R_{N}+h\right) & 0 & 0 \\
\tan \phi /\left(R_{N}+h\right) & 0 & 0
\end{array}\right]\left[\begin{array}{c}
\delta V_{x} \\
\delta V_{y} \\
\delta V_{z}
\end{array}\right]
$$

Where $\phi$ is the local geographic latitude angle, $R_{M}, R_{N}$ is the curvature of the earth radius. 


\section{Stationary Base Alignment Mode}

In the stationary alignment we usually suppose that the position is known and fixed. So, the values of velocity vector $V_{\mathrm{t}}^{\mathrm{n}}$ and angular velocity vector $\omega_{\mathrm{en}}^{\mathrm{n}}$ equal zero, then equations (29) and (30) can be written as:

$$
\begin{aligned}
& \delta \dot{V}=\left(\mathrm{C}_{\mathrm{n}}^{\mathrm{p}}-I\right) \mathrm{C}_{\mathrm{b}}^{\mathrm{n}} f^{b}+\hat{\mathrm{C}}_{\mathrm{b}}^{\mathrm{n}} \nabla^{b}-\delta \omega_{e n}^{\mathrm{n}} \times V_{\mathrm{t}}^{\mathrm{n}}-2 \Omega_{\mathrm{ie}}^{\mathrm{n}} \times \delta V \\
& \dot{\psi} \approx \omega_{\mathrm{np}}^{\mathrm{p}}=\left(I-\mathrm{C}_{\mathrm{n}}^{\mathrm{p}}\right) \Omega_{\mathrm{ie}}^{\mathrm{n}}+\delta \omega_{\mathrm{in}}^{\mathrm{n}}-\mathrm{C}_{\mathrm{b}}^{\mathrm{p}} \delta \omega_{\mathrm{ib}}^{\mathrm{b}}
\end{aligned}
$$

Where $\omega_{i n}^{n}=\omega_{e n}^{n}+\Omega_{i e}^{n}$.

The value of angular velocity of the earth and the specific forces in navigation frame can be written as:

$$
\begin{aligned}
\Omega_{i e}^{n} & =\left[\begin{array}{lll}
0 & \Omega \cos \phi & \Omega \sin \phi
\end{array}\right]^{T} \\
f^{\mathrm{n}} & =\left[\begin{array}{lll}
0 & 0 & \mathrm{~g}
\end{array}\right]^{T}
\end{aligned}
$$

Substituting equations $(33,34)$ into equations $(31,32)$ then the platform error model on the stationary base can be written as:

$$
\begin{aligned}
& \delta \dot{V}_{x}=-\psi_{y} \mathrm{~g}+2 \Omega \sin \phi \delta V_{y}+\nabla_{x}^{p} \\
& \delta \dot{V}_{y}=\psi_{x} \mathrm{~g}-2 \Omega \sin \phi \delta V_{x}+\nabla_{y}^{p} \\
& \dot{\psi}_{x}=-\sin \psi_{z} \Omega \cos \phi+\psi_{y} \Omega \sin \phi-\delta V_{y} /\left(R_{M}+h\right)+\varepsilon_{x}^{p} \\
& \dot{\psi}_{y}=\left(1-\cos \psi_{z}\right) \Omega \cos \phi-\psi_{x} \Omega \sin \phi+\delta V_{x} /\left(R_{N}+h\right)+\varepsilon_{y}^{p} \\
& \dot{\psi}_{z}=\left(\psi_{x} \cos \psi_{z}-\psi_{y} \sin \psi_{z}\right) \Omega \cos \phi+\delta V_{x} \tan \phi /\left(R_{N}+h\right)+\varepsilon_{z}^{p}
\end{aligned}
$$

\section{In-Flight Alignment Mode}

In-flight alignment, we usually get the INS velocity error equation from the acceleration measurement $\hat{f}^{p}$ then, from Eq. (5) we get:

$$
\begin{aligned}
\delta \dot{V}= & \left(I-\mathrm{C}_{\mathrm{p}}^{\mathrm{n}}\right) \hat{\mathrm{C}}_{\mathrm{b}}^{\mathrm{n}} \hat{f}^{b}+\mathrm{C}_{\mathrm{b}}^{\mathrm{n}} \nabla^{b}-\left(2 \delta \Omega_{\mathrm{ie}}^{\mathrm{n}}+\delta \omega_{\mathrm{en}}^{\mathrm{n}}\right) \\
& \times V_{\mathrm{t}}^{\mathrm{n}}-\left(2 \Omega_{\mathrm{ie}}^{\mathrm{n}}+\omega_{\mathrm{en}}^{\mathrm{n}}\right) \times \delta V+\delta g^{\mathrm{n}}
\end{aligned}
$$

The error model in-flight augmented with sensor errors can be written as:

$$
\left[\begin{array}{l}
\dot{x}_{1} \\
\dot{x}_{2}
\end{array}\right]=\left[\begin{array}{cc}
A(t) & I_{6 \times 6} \\
0_{6 \times 6} & 0_{6 \times 6}
\end{array}\right]\left[\begin{array}{l}
\mathrm{x}_{1} \\
\mathrm{x}_{2}
\end{array}\right]+\left[\begin{array}{c}
q(x, \mathrm{t}) \\
0_{6 \times 1}
\end{array}\right]
$$

A local level ENU (East-North-Up) frame is used as the navigation frame, vertical channel included. The state vectors consists of:

$$
\begin{aligned}
& x_{1}=\left[\begin{array}{llllll}
\delta \mathrm{V}_{x} & \delta \mathrm{V}_{y} & \delta \mathrm{V}_{z} & \psi_{x} & \psi_{y} & \psi_{z}
\end{array}\right] \\
& x_{2}=\left[\begin{array}{lllllll}
\nabla_{x} & \nabla_{y} & \nabla_{z} & \varepsilon_{\mathrm{x}}^{\mathrm{p}} & \varepsilon_{\mathrm{y}}^{\mathrm{p}} & \varepsilon_{\mathrm{z}}^{\mathrm{p}}
\end{array}\right]
\end{aligned}
$$

The linear part coefficient matrix $A(\mathrm{t})$ is the system dynamic matrix defined as [9], while the nonlinear part $\mathrm{q}(\mathrm{x}, t)$ can be computed as:

$$
q(x, \mathrm{t})=\left[\begin{array}{l}
\left(I-\mathrm{C}_{\mathrm{p}}^{\mathrm{n}}\right) \hat{f}^{p} \\
\left(I-\mathrm{C}_{\mathrm{n}}^{\mathrm{p}}\right) \omega_{i n}^{n}
\end{array}\right]
$$

System Jacobian matrix can be computed as: 


$$
\frac{\partial f(x, \mathrm{t})}{\partial x}=A(\mathrm{t})+\frac{\partial q(x, \mathrm{t})}{\partial x}
$$

\section{Filtering Mechanization}

In this section we simulate SINS large azimuth misalignment angle on both the stationary base and in-flight alignment by using nonlinear discretization, extended KALMAN filter and iterative filtering.

\section{V.1 Nonlinear discretization}

To be convenient, we write the nonlinear system states equation as:

$$
x(\mathrm{t})=f(x, \mathrm{t})+\mathrm{w}(\mathrm{t})
$$

Where $\boldsymbol{f}(\boldsymbol{x}, \boldsymbol{t})$ is the nonlinear function, $w(t)$ is the process noise. The variant matrix:

$$
\mathrm{Q}(\mathrm{t})=\mathrm{E}\left[\mathrm{w}(\mathrm{t}) \mathrm{w}^{T}(\mathrm{t})\right]
$$

Let $\Delta \mathrm{t}$ is the sampling time, and the solution of the scalar differential equation using Taylor expansion of $x(\mathrm{t}+\Delta \mathrm{t})$ :

$$
x(\mathrm{t}+\Delta \mathrm{t})=x(\mathrm{t})+f[x(\mathrm{t})] \Delta \mathrm{t}+\left.\frac{\partial f(x)}{\partial x}\right|_{\mathrm{x}=\mathrm{x}(t)} f[x(\mathrm{t})] \frac{(\Delta \mathrm{t})^{2}}{2}+\ldots . .
$$

Let $x_{k}=x(\mathrm{t}), x_{\mathrm{k}+1}=x(\mathrm{t}+\Delta \mathrm{t})$, ignore $2^{\text {nd }}$ order derivative term, the discretization equation can be written as:

$$
x_{\mathrm{k}+1}=x_{\mathrm{k}}+f\left(x_{\mathrm{k}}\right) \Delta \mathrm{t}+D\left(x_{k}\right) f\left(x_{\mathrm{k}}\right) \frac{(\Delta \mathrm{t})^{2}}{2}+\mathrm{w}_{\mathrm{k}}
$$

Where

$$
\begin{aligned}
& D\left(x_{k}\right)=\left.\frac{\partial f(x, \mathrm{t})}{\partial x}\right|_{\mathrm{x}=\mathrm{x}_{k}} \\
& \mathbf{w}_{k} \text { is the discretization process noise, and variant matrix } Q_{K}=\mathrm{E}\left[\mathrm{w}_{k} \mathrm{w}_{k}^{T}\right] \\
& Q_{k}=Q(\mathrm{t}) \cdot \Delta \mathrm{t}
\end{aligned}
$$

We use the two horizontal velocity error measurements, and then the measurement equation can be written as:

$$
\mathrm{z}_{\mathrm{k}}=H_{\mathrm{k}} x_{\mathrm{k}}+v_{\mathrm{k}}
$$

Where $\mathrm{v}_{k}$ is the measurement noise. Measurement matrix can be written as:

$$
H=\left[\begin{array}{ll}
I_{2 \times 2} & 0_{2 \times 8}
\end{array}\right]
$$

\section{V.2 Extended KALMAN Filter (EKF)}

To use extended KALMAN Filter we must linearize the nonlinear equation because the measurement equation is linear $[8,13]$. So, we only need linearized system equation. Let Eq. (43) can be rewritten as:

$$
x_{k+1}=F\left[x_{k}, \mathrm{k}\right]+\mathrm{w}_{k}
$$

Where

$$
\boldsymbol{F}\left[\boldsymbol{x}_{k}, \mathrm{k}\right]=\boldsymbol{x}_{k}+\boldsymbol{f}\left(x_{k}\right) \Delta \mathrm{t}+\boldsymbol{D}\left(x_{k}\right) \boldsymbol{f}\left(\boldsymbol{x}_{k}\right) \frac{\Delta \mathrm{t}^{2}}{2}
$$

Let $\Delta \mathrm{t}$ is small, the transition matrix with one step can be written as:

$$
\Phi(\mathrm{k}+1, \mathrm{k})=\left.\frac{\partial F[\boldsymbol{x}(\mathrm{k}), \mathrm{k}]}{\partial \boldsymbol{x}}\right|_{x=\dot{z} /(k / k)} \approx \boldsymbol{I}+\boldsymbol{D}[\hat{\boldsymbol{x}}(\mathrm{k} / \mathrm{k}] \Delta \mathrm{t}
$$


So, the extended Kalman Filter can be programmed as:

$$
\begin{aligned}
& \hat{\boldsymbol{x}}_{k+1 / k}= \hat{\boldsymbol{x}}_{k / k}+\boldsymbol{f}\left[\hat{\boldsymbol{x}}_{k / k}\right] \Delta \mathrm{t}+\boldsymbol{D}\left[\hat{\boldsymbol{x}}_{k / k}\right] \boldsymbol{f}\left[\hat{\boldsymbol{x}}_{k / k}\right] \Delta \mathrm{t}^{2} / 2 \\
& \hat{\boldsymbol{x}}_{k+1 / k+1}=\hat{\boldsymbol{x}}_{k+1 / k}+\boldsymbol{K}_{k+1}\left[z_{k+1}-\boldsymbol{H}_{k+1} \hat{\boldsymbol{x}}_{k+1 / k}\right] \\
& \boldsymbol{P}(\mathrm{k}+1 / \mathrm{k})=\Phi(\mathrm{k}+1, \mathrm{k}) \boldsymbol{P}(\mathrm{k} / \mathrm{k}) \Phi^{T}(\mathrm{k}+1, \mathrm{k})+\boldsymbol{Q}_{k} \\
& \boldsymbol{K}_{k+1}=\boldsymbol{P}_{k+1 / k} \boldsymbol{H}_{k+1}^{T}\left[\boldsymbol{H}_{k+1} \boldsymbol{P}_{k+1 / k} \boldsymbol{H}_{k+1}^{T}+\boldsymbol{R}_{k+1}\right]^{-1} \\
& \boldsymbol{P}_{\mathrm{k}+1 / \mathrm{k}+1}=\left[\boldsymbol{I}-\boldsymbol{K}_{\mathrm{k}+1} \boldsymbol{H}_{\mathrm{k}+1}\right] \boldsymbol{P}_{\mathrm{k}+1 / \mathrm{k}}\left[\boldsymbol{I}-\boldsymbol{K}_{k+1} \boldsymbol{H}_{k+1}\right]^{T} \\
&+ \\
&+\boldsymbol{K}_{\mathrm{k}+1} \boldsymbol{R}_{k+1} \boldsymbol{K}_{\mathrm{k}+1}^{\mathrm{T}}
\end{aligned}
$$

\section{V.3 Iterative filtering (IKF)}

$$
\begin{aligned}
\hat{\boldsymbol{x}}_{\mathrm{k} \mathrm{k}+1}= & \hat{\boldsymbol{x}}_{\mathrm{k} \mathrm{k}}+\boldsymbol{P}_{\mathrm{k} k} \Phi_{k+1 / k}^{T} \boldsymbol{P}_{\mathrm{k}+1 / \mathrm{k}}^{-1}\left[\hat{\boldsymbol{x}}_{\mathrm{k}+1 / \mathrm{k}+1}-\hat{\boldsymbol{x}}_{\mathrm{k} k+1}\right] \\
= & \hat{\boldsymbol{x}}_{\mathrm{k} \mathrm{k}}+\boldsymbol{P}_{\mathrm{k} k} \Phi_{\mathrm{k}+1 / k}^{\mathrm{T}} \boldsymbol{H}_{\mathrm{k}}^{\mathrm{T}}\left[\boldsymbol{H}_{\mathrm{k}+1} \boldsymbol{P}_{\mathrm{k}+1 \mathrm{k}} \boldsymbol{H}_{\mathrm{k}+1}^{\mathrm{T}}+\boldsymbol{R}_{k+1}\right]^{-1} \\
& {\left[z_{\mathrm{k}+1}-\boldsymbol{H}_{k+1} \hat{\boldsymbol{x}}_{\mathrm{k}+1 \mathrm{k}}\right] }
\end{aligned}
$$

The transition matrix with one step can be written as:

$$
\begin{aligned}
\Phi(\mathrm{k}+1, \mathrm{k})= & \left.\frac{\partial \boldsymbol{F}[\boldsymbol{x}(\mathrm{k}), \mathrm{k}]}{\partial \boldsymbol{x}}\right|_{\mathrm{x}=\dot{\mathrm{x}}(\mathrm{k} / \mathrm{k}+1)} \\
& \approx \boldsymbol{I}+\boldsymbol{D}[\hat{\boldsymbol{x}}(\mathrm{k} / \mathrm{k}+1] \Delta \mathrm{t}
\end{aligned}
$$

\section{Simulation Results}

A simulation program is carried out to validate the alignment accuracy associated with the error models. The following parameters are considered in the comparison study; the initial attitude angles are chosen equal zero; the constant and random biases of each accelerometer are chosen as $100 \mu \mathrm{g}$ and $5 \mu \mathrm{g}$ respectively, and the constant and random drifts of each gyro are chosen as $0.02^{\circ} / \mathrm{h}$ and $0.01^{\circ} / \mathrm{h}$ respectively. The measuring error of velocity is $0.1 \mathrm{~m} / \mathrm{s}$ and the system measured noise $\sigma_{\delta V}=.01 \mathrm{~m} / \mathrm{s}$. The local latitude of SINS place is $30^{\circ}$. The sampling time is chosen to be $50 \mathrm{msec}$. In the following two case studies are

\begin{tabular}{|c|c|c|c|c|}
\hline $\begin{array}{l}\text { Initial } \\
\text { Azimuth } \\
\text { [deg] }\end{array}$ & $\begin{array}{l}\text { Est. } \\
\text { Errot } \\
\text { angle } \\
\text { [sec] }\end{array}$ & EKF & IRFF & LKF \\
\hline \multirow[t]{3}{*}{10} & $\Delta \Psi$ & -254.2412 & -244.0982 & 215.1588 \\
\hline & $\Delta \theta$ & -21.2108 & -21.3302 & -255.0105 \\
\hline & $\Delta \gamma$ & 15.7242 & 15.7585 & 150.8116 \\
\hline \multirow[t]{3}{*}{15} & $\Delta \mathscr{W}$ & -223.7322 & -210.7751 & 1420.7 \\
\hline & $\Delta \theta$ & -23.0430 & -23.1915 & -394.900 \\
\hline & $\Delta \gamma$ & 14.6770 & 14.6699 & 138.300 \\
\hline \multirow[t]{3}{*}{20} & $\Delta \psi$ & -191.1677 & -176.0421 & 3661.5 \\
\hline & $\Delta \theta$ & -24.7919 & -24.9570 & -559.700 \\
\hline & $\Delta \gamma$ & 13.2539 & 13.1829 & 66.600 \\
\hline
\end{tabular}
demonstrated, the stationary alignment simulation and in-flight alignment simulation.

\section{Stationary alignment simulation}

The initial pitch and roll angle errors chosen as $1^{0}$, yaw error equal $20^{\circ}$, we used nonlinear error equation and small disturbance equation separately, alignment time equal $300 \mathrm{sec}$. We used nonlinear model and EKF, then the estimated error of the horizontal error angle and azimuth error angle are shown in Fig (1), and Fig (2) showing the estimated accelerometer biases and gyro drift. Table (1) shows the effect of the variation of initial large azimuth misalignment angle on the static attitude error by using the EKF, IKF, and linearized Kalman filter (LKF).

Table (1) 

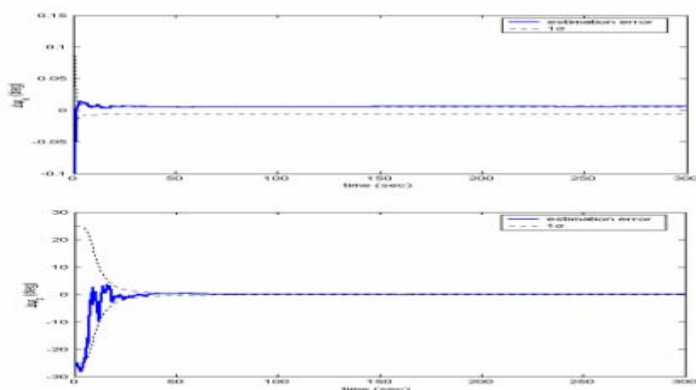

Fig (1) Estimated Attitude error angles
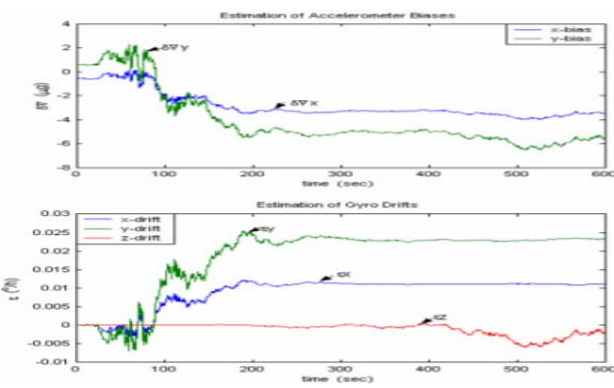

Fig (2) Estimated sensor bias and drift

\section{In-flight alignment Simulation}

From the From the trajectory data, under the assumption firstly, the missile flight is linear and secondly, with horizontal level maneuver with max heading variation chosen as $30^{\circ}$. The missile constant velocity $400 \mathrm{~m} / \mathrm{s}$, flight direction angle chosen as $60^{\circ}$, and the local latitude of SINS place is $30^{\circ}$. The initial pitch, roll angle errors chosen as $1^{\circ}$, and heading error chosen as $10^{\circ}$. The total time of alignment equal $600 \mathrm{sec}$. We used nonlinear error equation and small disturbance equation for in-flight alignment. Table (2) shown the static values of attitude error angles by using the different model of Kalman filters (EKF, IKF and LKF) in the case of linear flight and with turn maneuver.

Fig (3) shows the estimated errors of the horizontal error angle and azimuth error angle in the case of linear flight path. Fig (4) shows the estimated errors of the horizontal error angle and azimuth error angle in the turn maneuver. Fig $(5,6,7)$ and Fig $(8,9,10)$ shows the static values of attitude error angles in the linear flight and with level maneuver using EKF respectively.

\begin{tabular}{|l|l|l|l|l|}
\hline $\begin{array}{l}\text { In-flight } \\
\text { Cases }\end{array}$ & $\begin{array}{l}\text { Attitud } \\
\text { e error } \\
\text { [sec] }\end{array}$ & EKF & IKF & LKF \\
\hline \multirow{4}{*}{$\begin{array}{l}\text { Linear } \\
\text { Flight }\end{array}$} & $\Delta \psi$ & -185.319 & -140.9032 & 374.336 \\
\cline { 2 - 5 } & $\Delta \theta$ & -22.8078 & -23.8795 & -52.2318 \\
\cline { 2 - 5 } & $\Delta \gamma$ & 15.2366 & 15.0149 & 230.385 \\
\hline \multirow{4}{*}{$\begin{array}{l}\text { Turn } \\
\text { Maneuver }\end{array}$} & $\Delta \psi$ & -0.0633 & -0.6133 & 2164.6 \\
\cline { 2 - 5 } & $\Delta \theta$ & -0.1736 & -0.2888 & 501.400 \\
\cline { 2 - 5 } & $\Delta \gamma$ & -0.0040 & -0.1224 & 241.700 \\
\hline
\end{tabular}

Table (2) Static Attitude Error Angle
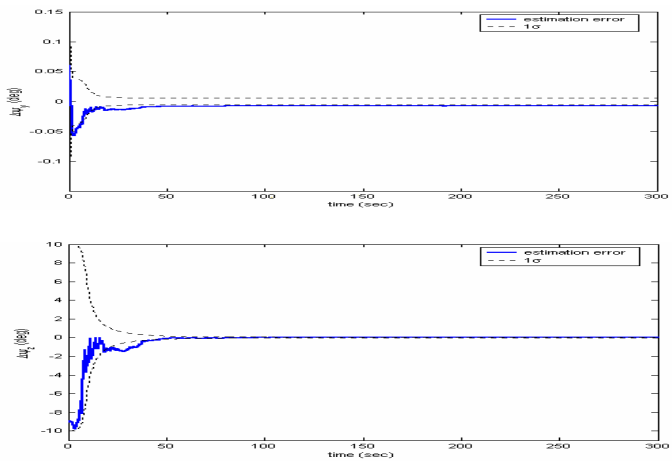

Fig (3) Estimated horizontal \& azimuth

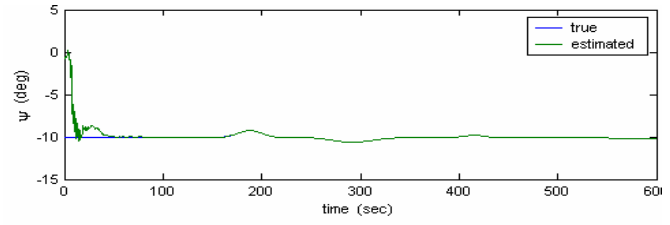

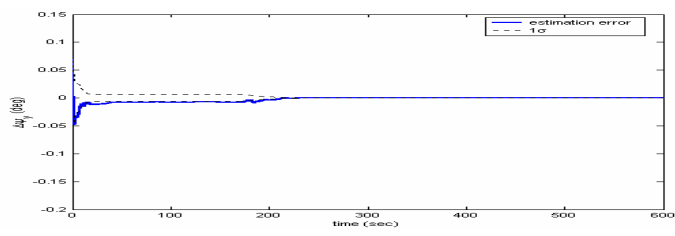

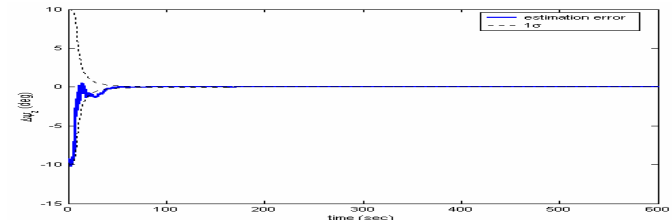

Fig (4) Estimated horizontal \& azimuth error angle with turn maneuver

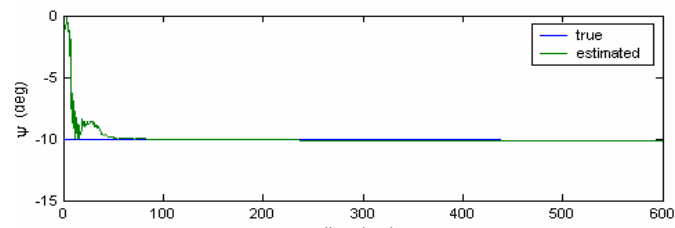



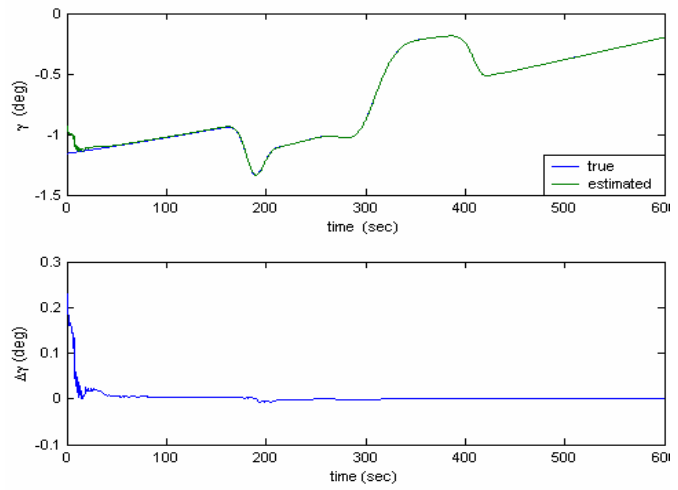

Fig (5) Estimated Attitude angle with linear flight
Fig (6) Estimated Attitude angle with turn maneuver

\section{Conclusion}

This paper presents a general nonlinear psi angles model that dose not require coarse alignment. In this model, the azimuth misalignment angle is assumed large. The velocity error model is also presented. In this paper SINS error model is presented, which can be used to design an alignment filter. Three different Kalman filter are designed based on the SINS nonlinear error model. The model presented was validated with a set of experimental results of stationary alignment and In-flight alignment using kinematics trajectory data to estimate all the parameters of inertial navigation system needed for the alignment and calibration. These results are helpful in design of stationary alignment process to improve the performance of the INS alignment during In-flight mode.

\section{References}

[1] Abraham Weinred and Itzhack Y. Bar-Itzhack, "The psi-angle error equation in strapdown inertial navigation systems", IEEE Aerospace and Electronic Systems, Vol. AES-14, No.3 May 1978, p539-p542. 
[2] Yu Jixing, "Kalman filter and its application in inertial navigation", Northwestern Polytechnical University Express, Xian, 1984.

[3] N.Loveren and J.K.Pieper, "A strapdown inertial navigation system for flat-earth model”, IEEE Aerospace and Electronic Systems, Vol. AES-33, No.1 January 1997, p214-p223.

[4] Itzhack Y. Bar-Itzhack and N. Berman, "Control theoretical approach to inertial navigation systems", Journal of Guidance vol.11 No.3, May-June 1988.

[5] Tuan Manh Pham, "Kalman filter mechanization for INS airstar", IEEE AES System Magazine, January 1992, p3-p11.

[6] Bruno M.Scherzinger, "Inertial navigation error models for large heading uncertainty", Proceeding of PLANS 1996 p477-p484.

[7] Xiaoying Kong, Eduardo Mario, and Hugh Durrant-Whyte, "Development of a non-linear psi-angle model for large misalignment errors and its application in INS alignment and calibration”, IEEE, international Conferences on Robotics and Automation, May 1999.

[8] Wei Chun ling, "Alignment of Inertial navigation and Terrain systems", PhD Thesis, Beijing University of Aeronautics and Astronaumatic, Beijing 100083, 2001.

[9] MYEONG-JONG YU and JANG GYU LEE "Comparison of SDINS In-Flight Alignment Using Equivalent Error Models”, IEEE Aerospace and Electronic Systems, Vol.-35, No.3 July 1999.

[10] Yu, M. J., Park, H. W., and Jeon, C. B. "Equivalent nonlinear error models of strapdown inertial navigation system", In proceeding of the AIAA 1997 GNC conference, Agu. 1997; AIAA paper 97-3563.

[11] Bruno M.Scherzinger and D.Blake Reid, and Mdified Strapdown; "Inertial navigation error models", Proceedings of PLANS 1994, p426-p430.

[12] S.P. Dmitriyev, O.A.Stepanov, and S.V.Shepel, "Nonlinear filtering methods application in INS alignment", IEEE Aerospace and Electronic Systems, Vol. AES-33, No.1 January 1997. P260-p271.

[13] S.J.julier, J.K.Uhlmann and H.F.Durrant-Whyte, "A New Approach for the Nonlinear Transformation of Means and Covariances in Linear Filters", IEEE Transactions on Automatic Control, 1996. 